
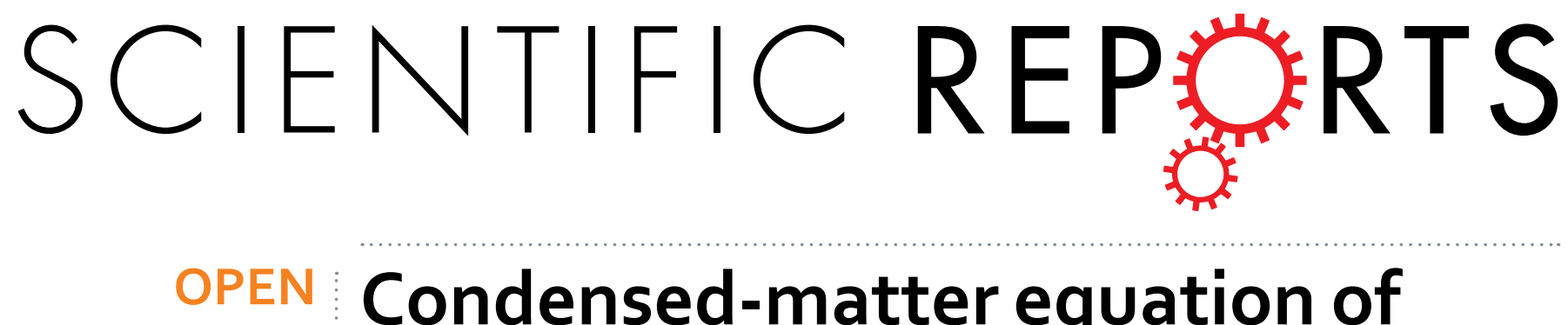

\title{
Condensed-matter equation of states covering a wide region of pressure studied experimentally
}

Received: 26 July 2016

Accepted: 18 November 2016

Published: 15 December 2016

\section{Elijah E. Gordon ${ }^{1}$, Jürgen Köhler² \& Myung-Hwan Whangbo}

The relationships among the pressure $P$, volume $V$, and temperature $T$ of solid-state materials are described by their equations of state (EOSs), which are often derived from the consideration of the finite-strain energy or the interatomic potential. These EOSs consist of typically three parameters to determine from experimental $P$ - $V$ - $T$ data by fitting analyses. In the empirical approach to EOSs, one either refines such fitting parameters or improves the mathematical functions to better simulate the experimental data. Despite over seven decades of studies on EOSs, none has been found to be accurate for all types of solids over the whole temperature and pressure ranges studied experimentally. Here we show that the simple empirical EOS, $P=\alpha_{1}(P V)+\alpha_{2}(P V)^{2}+\alpha_{3}(P V)^{3}$, in which the pressure $P$ is indirectly related to the volume $V$ through a cubic polynomial of the energy term $P V$ with three fitting parameters $\alpha_{1}-\alpha_{3}$, provides accurate descriptions for the $P$-vs- $V$ data of condensed matter in a wide region of pressure studied experimentally even in the presence of phase transitions.

One of the most important issues in condensed matter sciences, particularly, in geology and geophysics, is to accurately predict the structural and physical properties of solids under high pressure and temperature ${ }^{1-7}$. In general, a solid-state material under high pressure and temperature can exhibit properties quite different from those found at ambient conditions. At a given temperature $T$, a solid under external pressure $P$ decreases its volume $V$ with increasing $P$, but $V$ changes a lot more slowly than does $P$. The pressure-induced volume decrease may require a change in the structure type (i.e., the pattern of the relative atom arrangements in a repeat unit cell) thereby causing a structural phase transition and an associated physical property change. For example, when $P$ is increased at room temperature, elemental chalcogen $\mathrm{Te}^{8-14}, \mathrm{Se}^{15-20}$, or $\mathrm{S}^{15,21-25}$ undergoes a number of structural phase transitions while its electrical property changes from insulating at ambient pressure to metallic and superconducting at high pressure ${ }^{26,27}$. Hydrogen sulfide $\mathrm{H}_{2} \mathrm{~S}$ is a diamagnetic molecular species at ambient conditions, but is converted, under the pressure of over $\sim 110 \mathrm{GPa}$, to a condensed phase that becomes superconducting at $\sim$ $200 \mathrm{~K}^{25,28}$, the highest among all superconductors known so far. An isothermal EOS relates $P$ and $V$ at a certain temperature $T$. Over the past 70 years the $P$-vs- $V$ data have been studied for a variety of solids in various pressure ranges (e.g., see Table 1), and their EOSs have been examined. So far, however, no isothermal EOS is applicable to all types of solids and is accurate over the whole range of pressure studied especially when a solid undergoes several structural phase transitions in the pressure region studied.

With increasing pressure $P$, the volume $V$ of a solid under pressure changes very slowly compared with the pressure change. The shortcomings of the known EOSs originate essentially from the attempts to relate the fast changing variable $P$ to a very slowly changing variable $V$. These problems can be circumvented if the pressure change is related to a pressure-induced energy change that is associated with the volume $V$ and also varies nearly at the same rate as does $P$. The energy term $P V$ satisfies these two requirements because, while increasing $P$, the volume $V$ of a solid under pressure $P$ decreases very slowly so that the term $P V$ changes nearly as fast as $P$ in the entire range of $P$. Furthermore, at a given $P$, the term $P V$ is determined by the value of $V$, not by how the atoms are arranged within the volume so that the term $P V$ cannot be overly sensitive to phase transitions. With increasing $P$, the term $P V$ should increase slightly more slowly than does $P$ because $V$ decreases slightly under pressure. Therefore, it should be possible to accurately describe the $P$-vs- $V$ data of any solid over the entire pressure range studied experimentally by the simple EOS,

${ }^{1}$ Department of Chemistry, North Carolina State University, Raleigh, NC 27695-8204, USA. ${ }^{2}$ Max-Planck-Institut für Festkörperforschung, D-70569 Stuttgart, Germany. Correspondence and requests for materials should be addressed to M.-H.W. (email: mike_whangbo @ncsu.edu) 


\begin{tabular}{|c|c|c|}
\hline System & Pressure range (GPa) & Temperature (K) \\
\hline $\mathrm{Te}$ & $\begin{array}{c}0^{11}, 0-4^{10}, 4.5^{12}, 8^{13}, 4-36^{9}, 33^{14} \\
30-330^{8}\end{array}$ & 298 \\
\hline Se & $\begin{array}{c}0^{17}, 0-10^{15}, 4.6^{18}, 23^{19}, 28,{ }^{19} \\
87.9^{20}, 140^{19}, 5-150^{16}\end{array}$ & $293-298$ \\
\hline S & $\begin{array}{c}0^{22}, 0-30^{15}, 35-87^{15}, 89.4^{23} \\
145^{21}, 160^{24}, 173^{25}, 206.5^{21} \\
88-213^{21}\end{array}$ & $293-298$ \\
\hline Sn & $0-120^{\mathrm{a}-\mathrm{c}}$ & 298 \\
\hline $\mathrm{Au}$ & $4-70^{\mathrm{d}}$ & 298 \\
\hline $\mathrm{Cu}$ & $7-95^{\mathrm{e}}$ & 293 \\
\hline $\mathrm{LiF}$ & $0-4^{\mathrm{h}}, 1-9^{\mathrm{f}}, 0-30^{\mathrm{g}}$ & 298 \\
\hline $\mathrm{NaF}$ & $1-9^{\mathrm{f}}, 0-38^{\mathrm{g}}$ & 298 \\
\hline $\mathrm{NaCl}$ & $0-4^{\mathrm{i}}$ & 298 \\
\hline $\mathrm{CsCl}$ & $0-5^{\mathrm{k}}, 1-9^{\mathrm{f}}, 0-45^{\mathrm{j}}$ & 298,293 \\
\hline Ice VII & $3-19^{\mathrm{l}}, 4-128^{\mathrm{m}}$ & 300 \\
\hline $\mathrm{MgO}$ & $\begin{array}{c}0-8^{33}, 0-11^{30}, 0-20^{32}, 0-24^{31} \\
0-52^{29}, 4-120^{35}, 10-140^{34}\end{array}$ & 298 \\
\hline $\mathrm{MgSiO}_{3}$ & $\begin{array}{c}0-10^{40}, 0-20^{36}, 0-55^{37}, 36-83^{38} \\
29-91^{39}, 100-300^{41}\end{array}$ & 298 \\
\hline $\mathrm{Ar}$ & $0-2^{6, \mathrm{n}}$ & 40 \\
\hline $\mathrm{Kr}$ & $0-2^{6, n}$ & 60 \\
\hline $\mathrm{Xe}$ & $0-2^{6, \mathrm{n}}$ & 60 \\
\hline $\mathrm{H}_{2}$ & $0-2.6^{47}$ & 4.2 \\
\hline $\mathrm{D}_{2}$ & $0-2.6^{47}$ & 4.2 \\
\hline PCL & $0-0.2^{45}$ & 373.6 \\
\hline Liquid $\mathrm{H}_{2} \mathrm{O}$ & $0-0.1^{46}$ & 288 \\
\hline Liquid $\mathrm{H}_{2} \mathrm{O}$ & $0-0.1^{46}$ & 298 \\
\hline Liquid $\mathrm{H}_{2} \mathrm{O}$ & $0-0.1^{46}$ & 308 \\
\hline
\end{tabular}

Table 1. The pressure ranges (in GPa) and temperature (K) employed to examine the isothermal $P$-vs- $V$ relationships for various condensed matter ${ }^{\dagger, \ddagger}{ }^{\dagger}$ In our analysis for $S n$, the $\alpha-S n$ phase was excluded because it exists below $286 \mathrm{~K}$, but all other phases of $\mathrm{Sn}$ that exist at room temperature are included. ${ }^{\ddagger}$ For the references a-p, see Section 3 of the SI.

$$
P=\alpha_{1}(P V)+\alpha_{2}(P V)^{2}+\alpha_{3}(P V)^{3}+\cdots,
$$

which expands $P$ as a polynomial of $P V$, where the constants $\alpha_{i}(i=1,2,3$, etc.) are the fitting parameters. For those familiar with the traditional EOSs, use of Eq. 1 is quite unconventional because $P$ is expanded in terms of the variable $P V$ that contains itself. However, our goal is to find an accurate, though indirect, relationship between $P$ and $V$ valid for the entire pressure region studied experimentally by way of determining an accurate relationship between $P$ and $P V$. From the resulting $P$-vs- $V$ relationship, one can derive an accurate description of other thermodynamic quantity such as bulk modulus, as we demonstrated in our work. When this expression is truncated to $P=\alpha_{1}(P V)+\alpha_{2}(P V)^{2}, P=\alpha_{1}(P V)+\alpha_{2}(P V)^{2}+\alpha_{3}(P V)^{3}$, and $P=\alpha_{1}(P V)+\alpha_{2}(P V)^{2}+\alpha_{3}(P V)^{3}+\alpha_{4}(P V)^{4}$, we obtain the quadratic, cubic and quartic approximations for Eq. 1. Here we establish that the cubic approximation with three fitting parameters $\alpha_{1}-\alpha_{3}$ is accurate enough in describing the $P$-vs- $V$ data for condensed matter in a wide region of pressure experimentally probed even in the presence of several structural phase transitions.

\section{Results}

Formulation of the EOS. In testing whether an isothermal EOS is accurate over the entire range of pressure studied experimentally, the ideal systems to analyze would be elemental chalcogens Te, Se and S because they have been studied at room temperature over wide pressure ranges (i.e., 0-330 GPa for Te, 0-150 GPa for Se, and 0-213 GPa for S) (see Table 1), because each chalcogen undergoes a number of phase transitions with increasing $P$, and because their room-temperature atomic structures are known under widely different pressures. For each chalcogen, we begin our analysis by first determining the relative energies of its known atomic structures at various $P$ on the basis of density functional theory (DFT) calculations, the details of which are described in Methods. We summarize the space groups of the known atomic structures at various pressures $P$ (mostly around the room temperature), the volumes $V$ per atom, the energies $P V$ per atom, and the calculated electronic energies $E$ per atom in Section 1(a)-(c) of the supporting information (SI). The calculated electronic structures are also presented in terms of density of states (DOS) plots in Section 1(d)-(f) of the SI. As anticipated, with increasing pressure, the DOS plot for each chalcogen is shifted toward the higher energy while the band gap present at low pressure disappears at high pressure.

The calculated energy $E$ for Te is plotted as a function of $P$ in Fig. 1a (those for Se and $S$ in Section $2(a, b)$ of the SI), which shows a reasonable linear relationship, $E \approx a_{1} P+a_{0}$, with slope $a_{1}$ and intercept $a_{0}$. Fig. 1 a also plots 

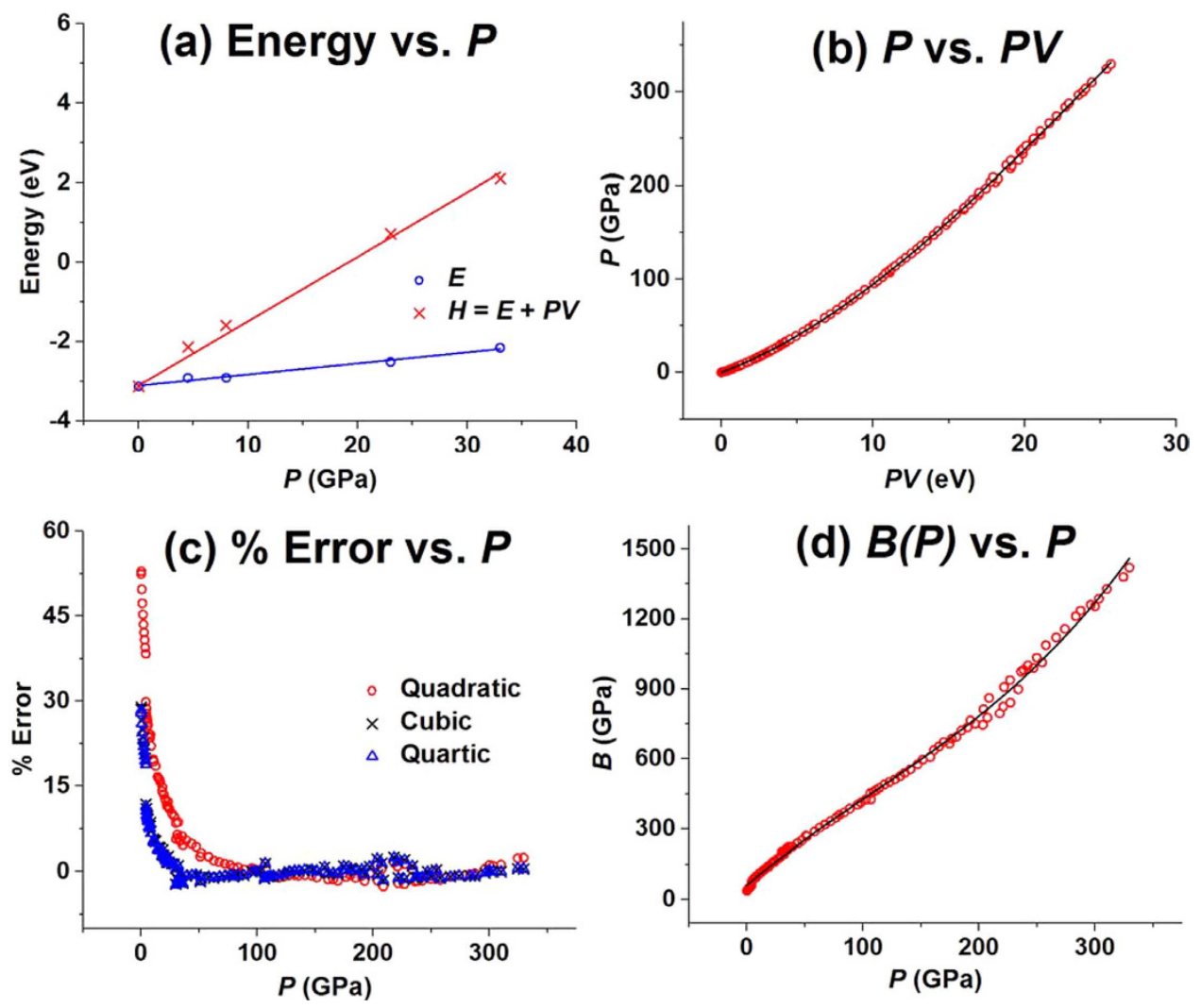

Figure 1. (a) The $E$-vs- $P$ and $H$-vs- $P$ plots calculated for Te, where $E, H$ and $P V$ are in eV units. The fitting coefficients $a_{1}, a_{0}$ and $b_{1}$ for the linear plots $E=a_{1} P+a_{0}$ and $H=b_{1} P+a_{0}$ are respectively $-3.0238,0.0246$ and 0.1589. (b) The $P$-vs- $P V$ plot constructed from the experimental $P$-vs- $V$ data for Te, where the solid line is the fitting curve obtained by using the cubic approximation for the EOS, Eq. 1. (c) The pressure-dependence of the $\%$ error, $100 \times\left(P_{\text {calc }}-P_{\text {expt }}\right) / P_{\text {expt }}$, of the $P$-vs- $P V$ plot obtained for Te by using the cubic approximation of the EOS, Eq. 1. (d) The pressure dependence of the bulk modulus $B(P)$ calculated for Te.

the enthalpy $H=E+P V$ per atom as a function of $P$ using $a_{0}$ as the intercept. This plot also exhibits a reasonable linear relationship, $H \approx b_{1} P+a_{0}$, with the slope $b_{1}>>a_{1}$ for all chalcogens. At a given temperature, therefore, the energy term $P V=H-E$ varies almost linearly with $P$, i.e., $P \approx P V /\left(b_{1}-a_{1}\right)$. Unlike the case of gaseous substances for which the $P V$ term is a constant independent of $P$ at a given $T$, the $P V$ term for each condensed-phase chalcogen increases almost linearly with $P$ because, compared with the rate of change in $P$, that in $V$ is very small. Nevertheless, the $H$-vs- $P$ plot for each chalcogen is slightly concave down with respect to the base line, $b_{1} P$. As already pointed out, this reflects that, with increasing $P$, the rate of change in $P$ is slightly greater than that in $P V$ due to a pressure-induced decrease in $V$. The latter allows one to expand $P$ as a power series of $P V$ as expressed in Eq. 1. Indeed, the $P$-vs- $V$ data points used for constructing the $H$-vs- $P$ plots are very well described, for example, by the quadratic approximation of Eq. 1 (see Section 2(c) of the SI). As will be discussed below, the nonlinear terms of Eq. 1, e.g., $\alpha_{2}(P V)^{2}$ and $\alpha_{3}(P V)^{3}$, are related to how the volume $V$ of a solid decreases under pressure $P$.

To test the applicability of the isothermal EOS, Eq. 1, in a wide region of pressure studied experimentally, we first analyze the experimental $P$-vs- $V$ data available in the literature for each chalcogen. The $P$-vs- $P V$ plot for Te, presented in Fig. 1b, reveals that the experimental points in the $0-330 \mathrm{GPa}$ region ${ }^{8-14}$ are very well described by the cubic approximation. The fitting curves from the quadratic and quartic approximations are not shown because they are practically impossible to distinguish, with naked eye alone, from that of the cubic approximation. The same conclusion is reached for $\mathrm{Se}^{15-20}$ and $\mathrm{S}^{15,21-25}$ (see Section 2(a,b) of the SI for Se and S, respectively). The fitting coefficients $\alpha_{1}-\alpha_{3}$ obtained for Te, Se and $S$ resulting from the cubic approximation are summarized in Table 2, and those from the quadratic, cubic and quartic approximations are compared in Section 2(d) of the SI. The coefficients $\alpha_{1}$ and $\alpha_{2}$ are always positive, and $\alpha_{1}>>\alpha_{2}>>\left|\alpha_{3}\right|$ with $\alpha_{2} / \alpha_{1} \approx 10^{-3}$ and $\left|\alpha_{3}\right| /\left|\alpha_{2}\right| \approx 10^{-4}$.

Error analysis. To assess the accuracies of the quadratic, cubic and quartic approximations for Eq. 1, we analyze the pressure-dependence of the absolute errors, $\Delta P=P_{\text {calc }}-P_{\text {expt }}$, as well as that of the \% errors, $100 \times \Delta$ $P / P_{\text {expt }}$, where $P_{\text {expt }}$ is the pressure observed experimentally, and $P_{\text {calc }}$ the one calculated from the fitting equations. The pressure-dependence of the \% errors for Te is shown in Fig. 1c, and those for Se and S in Section 2(a,b) of the SI. The maximum \% error is smaller than 5.5\% in the 10.9-330 GPa region for the cubic and quartic approximations, but smaller than $5.4 \%$ in the 38-330 GPa region for the quadratic approximation. The \% errors are large in the low $P$ region, but it should be pointed out that the associated absolute errors are rather small (for example, for $P_{\text {expt }}=0.98 \mathrm{GPa}$, the corresponding $P_{\text {calc }}$ values are $1.44,1.22$ and $1.22 \mathrm{GPa}$ from the quadratic, cubic and quartic 


\begin{tabular}{|c|c|c|c|c|c|}
\hline & $10^{2} \times \alpha_{1}$ & $10^{5} \times \alpha_{2}$ & $10^{9} \times \alpha_{3}$ & $P$ range & Max. \% error ${ }^{\mathrm{b}}$ \\
\hline \multicolumn{6}{|c|}{ (a) Solid-state condensed matter $\left(P\right.$ in GPa, and $V$ in $\left.\AA^{3}\right)$} \\
\hline $\mathrm{Te}$ & 3.785 & 1.501 & -1.130 & $0-330$ & $\begin{array}{l}5.5(P>10.9) \\
29(P<10.9)\end{array}$ \\
\hline $\mathrm{Se}$ & 4.782 & 2.421 & +1.325 & $0-150$ & $\begin{array}{l}3.6(P>23) \\
44(P<23)\end{array}$ \\
\hline $\mathrm{S}^{\mathrm{a}}$ & 3.587 & 7.987 & -16.20 & $0-213$ & $\begin{array}{l}4.6(P>36) \\
13(P<36)\end{array}$ \\
\hline Sn & 3.978 & 1.897 & -2.039 & $0-120$ & $\begin{array}{l}2.2(P>10.3) \\
8.0(P<10.3)\end{array}$ \\
\hline $\mathrm{Au}$ & 5.839 & 2.079 & -4.474 & $4-70$ & 1.9 \\
\hline $\mathrm{Cu}$ & 8.543 & 4.582 & -10.02 & $7-95$ & $\begin{array}{l}1.0(P>56) \\
6.5(P<56)\end{array}$ \\
\hline LiF & 6.064 & 6.646 & -59.75 & $0-30$ & 0.98 \\
\hline $\mathrm{NaF}$ & 4.068 & 2.922 & -13.88 & $0-38$ & 1.5 \\
\hline $\mathrm{NaCl}$ & 2.232 & 2.006 & -11.35 & $0-4$ & 0.1 \\
\hline $\mathrm{CsCl}$ & 1.518 & 0.6655 & -0.8938 & $0-45$ & 6.4 \\
\hline Ice VII & 4.898 & 8.732 & -24.99 & $3-128$ & 3.0 \\
\hline $\mathrm{MgO}$ & 5.379 & 1.666 & -1.192 & $0-142$ & 0.6 \\
\hline $\mathrm{MgSiO}_{3}$ & 2.437 & 0.2434 & -0.04751 & $0-265$ & 1.2 \\
\hline $\mathrm{Ar}$ & 2.682 & 18.55 & -876.3 & $0-2$ & 2.5 \\
\hline $\mathrm{Kr}$ & 2.198 & 12.04 & -459.7 & $0-2$ & 2.2 \\
\hline $\mathrm{Xe}$ & 1.728 & 6.493 & -175.2 & $0-2$ & 1.6 \\
\hline $\mathrm{H}_{2}$ & 3.323 & 104.0 & -7594 & $0-2.6$ & $\begin{array}{l}3.0(P>0.34) \\
26(P<0.34)\end{array}$ \\
\hline $\mathrm{D}_{2}$ & 3.552 & 99.32 & -7221 & $0-2.6$ & $\begin{array}{c}2.0(P>0.15) \\
18(P<0.15)\end{array}$ \\
\hline \multicolumn{6}{|c|}{ (b) Non-solid-state condensed matter ( $P$ in bar, and $V$ in $\left.\AA^{3}\right)$} \\
\hline PCL & 1.03976 & 6.290 & -7.651 & $0-2000$ & 0.3 \\
\hline $\mathrm{H}_{2} \mathrm{O}, 15^{\circ} \mathrm{C}$ & 0.03343 & $5.222 \times 10^{-3}$ & $-1.073 \times 10^{-4}$ & $0-1000$ & 0 \\
\hline $\mathrm{H}_{2} \mathrm{O}, 25^{\circ} \mathrm{C}$ & 0.03336 & $5.026 \times 10^{-3}$ & $-9.835 \times 10^{-5}$ & $0-1000$ & 0 \\
\hline $\mathrm{H}_{2} \mathrm{O}, 35^{\circ} \mathrm{C}$ & 0.03326 & $4.901 \times 10^{-3}$ & $-9.451 \times 10^{-5}$ & $0-1000$ & 0 \\
\hline
\end{tabular}

Table 2. The coefficients $\alpha_{1}-\alpha_{3}$ of the isothermal EOS, $P=\alpha_{1}(P V)+\alpha_{2}(P V)^{2}+\alpha_{3}(P V)^{3}$, obtained for various condensed matter. The range of the pressure $P$ used for each fitting analysis and the maximum $\%$ error found in the pressure range are also given ${ }^{\mathrm{a}, \mathrm{b}}$. ${ }^{\mathrm{a}}$ The $P$-vs- $V$ data points of the metastable S-III phase between 3-58 GPa (obtained by quenching) reported in ref. 15 were not included in the $P$-vs- $P V$ plot. However, including them does not change the quality of the fitting analysis. ${ }^{b}$ Unless mentioned otherwise, the maximum $\%$ error refers to the entire pressure region studied.

approximations, respectively) (see Section 2(e) of the SI). In general, the cubic and quartic approximations are similar in accuracy, and are more accurate than the quadratic approximation especially in the low $P$ region. A similar conclusion is reached from the \% error and absolute error plots calculated for Se in the 0-140 GPa range and for $\mathrm{S}$ in the $0-213 \mathrm{GPa}$ range (see Section $2(\mathrm{a}, \mathrm{b})$ of the SI).

Applicability to other condensed matter. The above analyses of the experimental $P$-vs- $V$ data for elemental chalcogens suggest strongly that the cubic approximation of the isothermal EOS, Eq. 1, can accurately describe the experimental $P$-vs- $V$ data for various solids in the whole pressure range studied experimentally. To establish this point, we examine the experimental $P$-vs- $V$ data for various solid-state condensed matter listed in Table 1, which include the elemental $\mathrm{Sn}$, the transition-metals $\mathrm{Au}$ and $\mathrm{Cu}$, the alkali halides $\mathrm{LiF}, \mathrm{NaF}, \mathrm{NaCl}$ and $\mathrm{CsCl}$, ice VII, the oxides $\mathrm{MgO}$ and $\mathrm{MgSiO}_{3}$, the noble gases $\mathrm{Ar}$, $\mathrm{Kr}$ and $\mathrm{Xe}$, as well as molecular hydrogen $\mathrm{H}_{2}$ and $\mathrm{D}_{2}$. As representative examples of these analyses, we discuss the oxides $\mathrm{MgO}^{29-35}$ and $\mathrm{MgSiO}_{3}{ }^{36-41}$. The isothermal $P$-vs- $V$ relationships of these oxides have been extensively studied because they are the end members of $(\mathrm{Mg}, \mathrm{Fe})$ $\mathrm{O}^{42,43}$ and $(\mathrm{Mg}, \mathrm{Fe}) \mathrm{SiO}_{3}$ perovskite ${ }^{42,44}$, which are the important components of the Earth's lower mantle. The $P$-vs- $V$ relationships for $\mathrm{MgO}^{29-35}$ were examined at room temperature in the $0-140 \mathrm{GPa}$ range, and those for $\mathrm{MgSiO}_{3}{ }^{36-41}$ at room temperature in the $0-300 \mathrm{GPa}$ range. The $P$-vs- $P V$ plots and the $\%$ error vs. $P$ plots for $\mathrm{MgO}$ and $\mathrm{MgSiO}_{3}$ are presented in Fig. 2. The maximum $\%$ error is smaller than $\sim 0.6 \%$ for $\mathrm{MgO}$, and smaller than $\sim 1 \%$ for $\mathrm{MgSiO}_{3}$, in the entire pressure ranges studied experimentally. For the remainder of the solids listed in Table 1, our results are summarized in Section 3(a)-(k) of the SI. The fitting coefficients $\alpha_{1}-\alpha_{3}$ obtained for the solid-state condensed matter of Table 1 from the cubic approximation are listed in Table 2 together with the maximum \% errors. It is clear that the cubic approximation of the isothermal EOS, Eq. 1, provides an accurate description in the entire pressure regions examined experimentally. (Hereafter, the cubic approximation of Eq. 1 will be used without further mentioning).

The isothermal EOS, Eq. 1, is also applicable to non-solid-state condensed matter. As examples, we analyze the experimental $P$-vs- $V$ data for the polymer, poly $\left(\varepsilon\right.$-caprolactone) $(\mathrm{PCL})^{45}$, determined at $100.6^{\circ} \mathrm{C}$ in the $0-0.2 \mathrm{GPa}$ region as well as those for liquid $\mathrm{H}_{2} \mathrm{O}^{46}$ determined at $15^{\circ} \mathrm{C}, 25^{\circ} \mathrm{C}$ and $35^{\circ} \mathrm{C}$ in the $0-0.1 \mathrm{GPa}$ region. Our results 

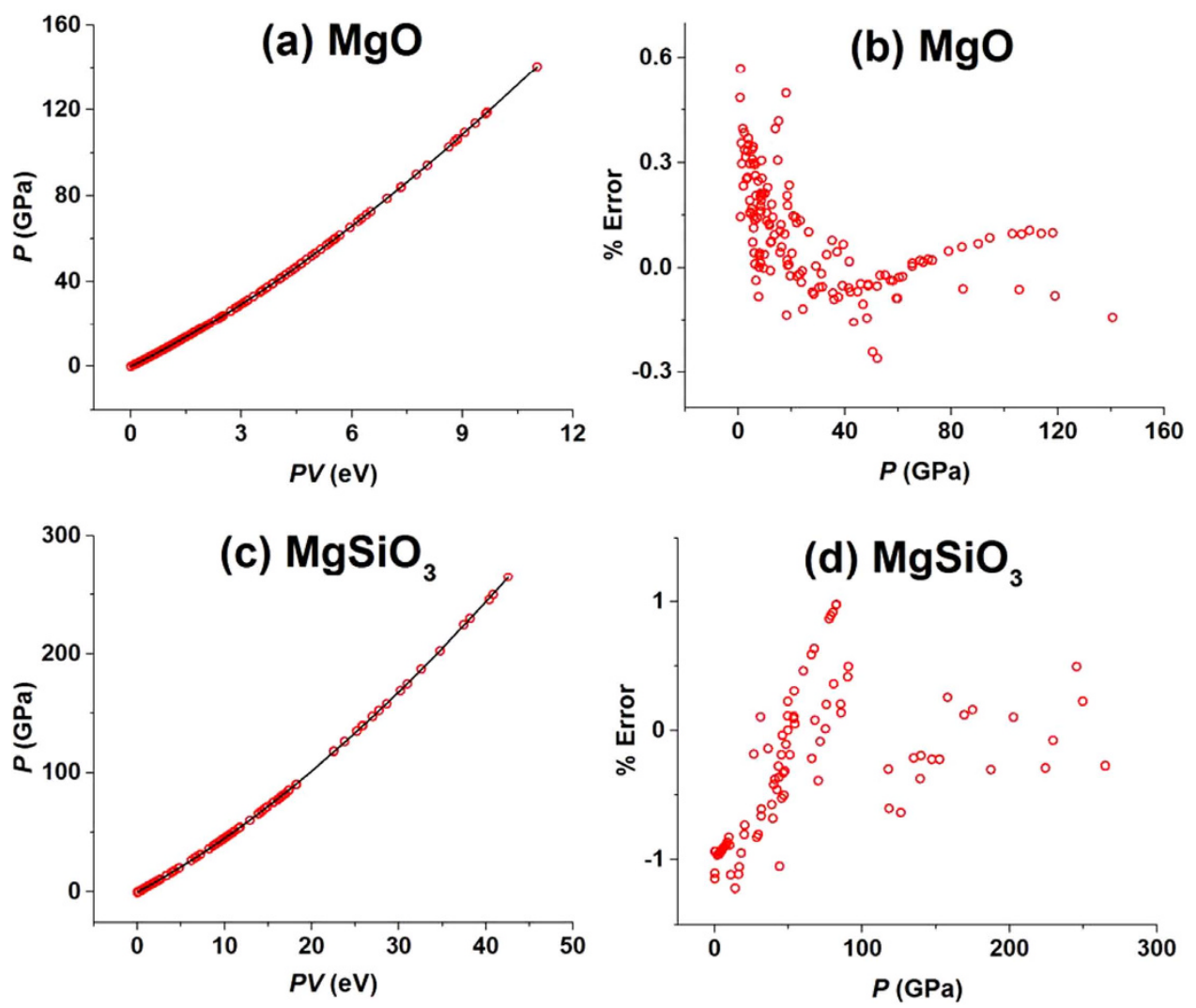

Figure 2. (a,c) The $P$-vs- $P V$ plots constructed from the experimental $P$-vs- $V$ data for $\mathrm{MgO}$ and $\mathrm{MgSiO}_{3}$, respectively, where the solid lines are the fitting curves obtained by using the cubic approximation of the EOS, Eq. 1. (b,d) The plots of the \% errors vs. pressure obtained for $\mathrm{MgO}$ and $\mathrm{MgSiO}_{3}$, respectively, by using the cubic approximation of the EOS, Eq. 1.

summarized in Section 3(m)-(p) of the SI show that the maximum \% error for the polymer is smaller than $0.3 \%$, and that for liquid water is practically zero, in the entire pressure region studied. Clearly, Eq. 1, provides an accurate description of the $P$-vs- $V$ relationship for these materials. It should be pointed out that the ideal gas law is a special case of Eq. 1, when the PV term is a constant independent of $P$.

\section{Discussion}

Bulk modulus. Now that Eq. 1 provides an isothermal EOS accurate for the entire pressure range studied for a given system, we search for a simple expression for the corresponding bulk modulus $B$ valid for the entire pressure region. In the cubic approximation, Eq. 1 is a quadratic equation of $P$, from which $P$ is written in terms of $V$ as

$$
P=-\left(\frac{\alpha_{2}}{2 \alpha_{3}}\right)\left(\frac{1}{V}\right)+\left(\frac{\sqrt{\left(\alpha_{2}^{2}-4 \alpha_{1} \alpha_{3}\right) V^{2}+4 \alpha_{3} V}}{2 \alpha_{3} V^{2}}\right)
$$

Thus, the bulk modulus $B$ is expressed as

$$
\begin{aligned}
B=-V\left(\frac{\partial P}{\partial V}\right)_{T}= & -\left(\frac{\alpha_{2}}{2 \alpha_{3} V}\right)-\left(\frac{1}{2 \alpha_{3} V}\right)\left(\frac{\left(\alpha_{2}{ }^{2}-4 \alpha_{1} \alpha_{3}\right) V+2 \alpha_{3}}{\sqrt{\left(\alpha_{2}{ }^{2}-4 \alpha_{1} \alpha_{3}\right) V^{2}+4 \alpha_{3} V}}\right) . \\
& +\left(\sqrt{\left(\alpha_{2}{ }^{2}-4 \alpha_{1} \alpha_{3}\right) V^{2}+4 \alpha_{3} V}\right)\left(\frac{1}{\alpha_{3} V^{2}}\right)
\end{aligned}
$$

This equation expresses the bulk modulus as a function of volume, namely, $B(V)$. For each value of $V$, however, there is a unique value of $P$ associated with it, so that the $B(V)$ vs. $V$ relationship can be easily converted to the corresponding $B(P)$ vs. $P$ relationship. For convenient use of this relationship, we fit the $B(P)$-vs. $-P$ relationship by the polynomial,

$$
B(P)=B_{0}+B_{1} P+B_{2} P^{2}+B_{3} P^{3} .
$$




\begin{tabular}{|c|c|c|c|c|c|c|}
\hline & $B_{0, \text { expt }}$ & $B_{0, \text { calc }}$ & $B_{0}$ & $B_{1}$ & $B_{2}$ & $B_{3}$ \\
\hline $\mathrm{Te}$ & $24(\mathrm{P}=2)^{14}$ & 37.68 & 62.48 & 4.135 & $-7.200 \times 10^{-3}$ & $2.278 \times 10^{-5}$ \\
\hline $\mathrm{Se}$ & $48.1(\mathrm{P}=7.7)^{18}$ & 28.09 & 46.99 & 5.311 & $-4.021 \times 10^{-2}$ & $1.516 \times 10^{-4}$ \\
\hline S & $14.5^{21}$ & 20.10 & 25.28 & 2.575 & $-2.635 \times 10^{-4}$ & $2.837 \times 10^{-5}$ \\
\hline Sn & $54.6^{\circ}, 55.4^{\circ}, 54.92^{\mathrm{p}}$ & 64.31 & 59.58 & 5.262 & $-4.100 \times 10^{-2}$ & $2.461 \times 10^{-4}$ \\
\hline $\mathrm{Au}$ & $166.65^{6, d}$ & $\begin{array}{c}192.09 \\
(\mathrm{P}=4.42)\end{array}$ & 171.09 & 3.516 & $2.146 \times 10^{-2}$ & $-2.613 \times 10^{-5}$ \\
\hline $\mathrm{Cu}$ & $133^{6, q}$ & $\begin{array}{c}184.48 \\
(\mathrm{P}=7.2)\end{array}$ & 156.32 & 4.025 & $-4.760 \times 10^{-3}$ & $6.803 \times 10^{-5}$ \\
\hline $\mathrm{LiF}$ & $66.4^{6, q}$ & 57.94 & 58.06 & 4.136 & $1.520 \times 10^{-2}$ & $4.610 \times 10^{-3}$ \\
\hline $\mathrm{NaF}$ & $46.1^{6, q}$ & 53.20 & 52.44 & 5.685 & -0.1005 & $3.900 \times 10^{-3}$ \\
\hline $\mathrm{NaCl}$ & $23.5^{6}$ & $\begin{array}{c}25.20 \\
(\mathrm{P}=0.106)\end{array}$ & 24.76 & 4.440 & $-8.684 \times 10^{-2}$ & $2.592 \times 10^{-2}$ \\
\hline $\mathrm{CsCl}$ & $16.8^{6, \mathrm{q}}$ & 27.57 & 28.79 & 4.896 & $-7.036 \times 10^{-2}$ & $1.290 \times 10^{-3}$ \\
\hline Ice VII & $23.9^{6, \mathrm{q}}$ & $\begin{array}{c}38.71 \\
(\mathrm{P}=3.16)\end{array}$ & 30.37 & 2.980 & $-1.950 \times 10^{-3}$ & $7.809 \times 10^{-5}$ \\
\hline $\mathrm{MgO}$ & $153-182^{30}$ & 170.66 & 171.63 & 3.562 & $-4.270 \times 10^{-3}$ & $2.269 \times 10^{-5}$ \\
\hline $\mathrm{MgSiO}_{3}$ & $200-340^{41}$ & 253.84 & 255.85 & 2.995 & $1.370 \times 10^{-3}$ & $2.150 \times 10^{-6}$ \\
\hline $\mathrm{Ar}$ & $2.35^{6, \mathrm{n}}$ & 3.49 & 3.482 & 6.053 & -1.732 & 0.9499 \\
\hline $\mathrm{Kr}$ & $2.49^{6}$ & 3.66 & 3.658 & 5.909 & -1.514 & 0.8780 \\
\hline $\mathrm{Xe}$ & $3.02^{6}$ & $4.52(\mathrm{P}=0.05)$ & 4.218 & 5.942 & -1.373 & 0.7384 \\
\hline $\mathrm{H}_{2}$ & $0.170^{51}, 0.174^{52}$ & 0.44 & 0.5707 & 5.002 & -1.521 & 0.5286 \\
\hline $\mathrm{D}_{2}$ & $0.315^{51}, 0.337^{52}$ & 0.70 & 0.7980 & 4.979 & -1.427 & 0.4953 \\
\hline PCL & $3.01^{45}$ & 1.683 & 1.679 & 8.491 & -7.773 & 86.34 \\
\hline $\mathrm{H}_{2} \mathrm{O}\left(15^{\circ} \mathrm{C}\right)$ & $2.140^{46}$ & 2.138 & 2.138 & 5.627 & 2.734 & 4.710 \\
\hline $\mathrm{H}_{2} \mathrm{O}\left(25^{\circ} \mathrm{C}\right)$ & $2.210^{46}$ & 2.213 & 2.213 & 5.605 & 2.388 & 5.164 \\
\hline $\mathrm{H}_{2} \mathrm{O}\left(35^{\circ} \mathrm{C}\right)$ & $2.250^{46}$ & 2.256 & 2.256 & 5.632 & 2.277 & 5.649 \\
\hline
\end{tabular}

Table 3. The coefficients of the bulk modulus formulas, $B(P)=B_{0}+B_{1} P+B_{2} P^{2}+B_{3} P^{3}$, obtained for various condensed matter using $\boldsymbol{P}$ and $\boldsymbol{B}(\boldsymbol{P})$ in GPa units. For comparison, the $B_{0, \mathrm{expt}}$ and $B_{0 \text {,calc }}$ values are also listed (see the text for the definition) ${ }^{\mathrm{a}, \mathrm{b}}$. ${ }^{\mathrm{a}}$ For the references $\mathrm{d}, \mathrm{l}$, and $\mathrm{n}-\mathrm{q}$, see Section 3 of the SI. ${ }^{\mathrm{b}}$ Unless otherwise stated, the $B_{0, \text { expt }}$ and $B_{0, \text { calc }}$ refer to the values at $P=0$. When these values are obtained at a nonzero $P$, the value of $P$ is specified in the parenthesis.

The $B(P)$-vs- $P$ plot thus-obtained for Te is presented in Fig. $1 \mathrm{~d}$. For other solids listed in Table 1, the $B(P)$-vs- $P$ plots are presented in Section $3(\mathrm{a})-(\mathrm{r})$ of the SI. The coefficients $B_{0}, B_{1}, B_{2}$ and $B_{3}$ determined for each condensed matter are summarized in Table 3 , which also lists the calculated bulk modulus at $P=0$, referred to as $B_{0, \text { calc }}$, for each system. The $B_{0}$ deviates from the $B_{0 \text {,calc }}$ because the polynomial fitting (Eq. 4 ) poorly describe the low-pressure region. Nevertheless, the $B_{0}$ and $B_{0 \text {,calc }}$ values are quite similar for all systems except for Te and Se.

For every system, our analysis leads to only one $B_{0}$ value because the entire pressure region studied is represented by the single fitting curve, Eq. 4 . In the traditional study for a system undergoing several phase transitions, each phase covering a certain pressure region (say, $P_{1}$ to $P_{2}$ ) is described by the EOS covering only the pressure region $P_{1}-P_{2}$. The resulting EOS for each different phase generates the bulk modulus, which we will refer to as $B_{0 \text { expt }}$, and the virtual volume $V_{0}$. It is the $B_{0 \text {,expt }}$ obtained for the "first" phase (i.e., the phase stable in the lowest pressure region for which $P_{1}=0$ ) that should be compared with the $B_{0}$ or the $B_{0 \text {,calc }}$ value obtained from our EOS. For systems with several phases, Table 3 lists only the $B_{0 \text {,expt }}$ values of their first phases. Clearly, these $B_{0, \text { expt }}$ values are well described by the $B_{0}$ and/or $B_{0 \text {,calc }}$ values determined from our EOS analyses. The $B_{0 \text {,expt }}$ values found for the various phases of Te, Se and S can be accounted for in terms of our EOS analyses as presented in Section 4 of the SI.

Qualitative meaning of the EOS. To gain insight into the meaning of the EOS, Eq. 1, we rewrite it in a slightly different form. In general, $\alpha_{1}(P V)>>\alpha_{2}(P V)^{2}>>\alpha_{3}(P V)^{3}$ so that $P \approx \alpha_{1}(P V)$. By using this approximation, Eq. 1 is rewritten as

$$
V \approx \frac{1}{\alpha_{1}}\left(1-\frac{\alpha_{2}}{\alpha_{1}^{2}} P-\frac{\alpha_{3}}{\alpha_{1}^{3}} P^{2}\right)=V_{c}\left(1-\alpha_{2} V_{c}^{2} P-\alpha_{3} V_{c}^{3} P^{2}\right)
$$

where the volume $V_{\mathrm{c}}$ is defined as $V_{\mathrm{c}} \equiv 1 / \alpha_{1}$, which is very close to the volume at zero-pressure, $V_{0}$. Eq. 5 reveals that the decrease in the volume of a condensed matter under pressure is a polynomial function of $P$. Since $\alpha_{2}\left(P V_{\mathrm{c}}\right)^{2}>>\left|\alpha_{3}\right|\left(P V_{\mathrm{c}}\right)^{3}$, the term $\alpha_{2} V_{\mathrm{c}}^{2} P$ dominates over $\alpha_{3} V_{\mathrm{c}}^{3} P^{2}$. Namely, the volume decreases with increasing $P$. The $\alpha_{3} V_{c}^{3} P^{2}$ term compensates the overcorrection (when $\alpha_{3}<0$ ), or the under-correction (when $\alpha_{3}>0$ ), given by the $\alpha_{2} V_{c}^{2} P$ term. In essence, the EOS, Eq. 1, reveals that the volume of a solid under pressure can be described as a polynomial function of pressure. 
Summary. We presented the empirical equation of state that accurately describes the pressure-versus-volume data for various types of condensed matter in a wide region of pressure studied experimentally. This is also true for systems undergoing several phase transitions in the pressure region studied. This equation of states results from the fact that the pressure change of a condensed matter is accurately described by a cubic polynomial of the pressure times the volume.

\section{Methods}

Our non-spin-polarized DFT calculations employed the frozen-core projector augmented wave method ${ }^{47,48}$ encoded in the Vienna ab initio simulation package ${ }^{49}$, and the generalized-gradient approximation of Perdew, Burke and Ernzerhof ${ }^{50}$ for the exchange-correlation functional. To ensure the accuracies of the calculations, a high plane-wave cut-off energy of $1000 \mathrm{eV}$ was used, and the Brillouin zone associated with each repeat unit cell was sampled by a large number of k-points. For example, the $R \overline{3} m$ structure of $\mathrm{S}$ at $206 \mathrm{GPa}$ was calculated by using a set of $24 \times 24 \times 24 \mathrm{k}$-points. Our calculations for Te, Se and S employed their reported crystal structures under various pressures, except for the 160 and $173 \mathrm{GPa}$ structures of $\mathrm{S}$ as described in Section $1(\mathrm{c})$. The threshold for the self-consistent-field energy convergence was set at $10^{-8} \mathrm{eV}$ for all structures of Se, Te and S. For the optimization of the structures of $\mathrm{S}$ at 160 and $173 \mathrm{GPa}$, the threshold for the force convergence at each atom was set at $0.005 \mathrm{eV} / \AA$.

\section{References}

1. Birch, F. Finite Elastic Strain of Cubic Crystals. Phys. Rev. 71, 809-824 (1947).

2. Vinet, P., Ferrante, J., Smith, J. R. \& Rose, J. H. A universal equation of state for solids. J. Phys. C: Solid State Phys. 19, L467-L473 (1986).

3. For a recent review, see: Garai, J. Semiempirical pressure-volume-temperature equation of state: $\mathrm{MgSiO}_{3}$ perovskite is an example. J. Appl. Phys. 102, 123506 (2007).

4. Cohen, R. E., Gülsren, O. \& Hemley, R. J. Accuracy of equation-of-state formulations. Am. Mineral. 85, 338-344 (2000).

5. Roy, S. B. \& Roy, P. B. Applicability of isothermal three-parameter equations of state of solids-a reappraisal. J. Phys.: Condens. Matter 17, 6193-6216(2005).

6. Taravillo, M., Baonza, V. G., Núñez, J. \& Cáceres, M. Simple equation of state for solids under compression. Phys. Rev. B 54, 7034-7045 (1996).

7. Anderson, O. L. Equations of State of Solids for Geophysics and Ceramic Science (Oxford University Press, 1994).

8. Sugimoto, T. et al. Bcc-fcc structure transition of Te. J. Phys. Conf. Ser. 500, 192018 (2014).

9. Hejny, C. \& McMahon, M. I. Large structural modulations in incommensurate Te-III and Se-IV. Phys. Rev. Lett. 91,215502 (2003).

10. Vaidya, S. N. \& Kennedy, G. C. Compressibility of 22 elemental solids to 45 kB. J. Phys. Chem. Solids 33, 1377-1389 (1972).

11. Adenis, C., Langer, V. \& Lindqvist, O. Reinvestigation of the structure of tellurium. Acta Cryst. C 45, 941-942 (1989).

12. Aoki, K., Shimomura, O. \& Minomura, S. Crystal structure of the high-pressure phase of tellurium. J. Phys. Soc. Jpn. 48, 551-556 (1980).

13. Takumi, M., Masamitsu, T. \& Nagata, K. X-ray structural analysis of the high-pressure phase III of tellurium. J. Phys.: Condens. Matter 14, 10609-10613 (2002).

14. Parthasarathy, G. \& Holzapfel, W. B. High-pressure structural phase transitions in tellurium. Phys. Rev. B 37, 8499-8501 (1988)

15. Degtyareva, O. et al. Novel chain structures in group VI elements. Nat. Mater. 4, 152-155 (2005).

16. Akahama, Y., Kobayashi, M. \& Kawamura, H. Structural studies of pressure-induced phase transitions in selenium up to $150 \mathrm{GPa}$. Phys. Rev. B 47, 20-26 (1993).

17. Marsh, R. E. \& Pauling, L. The crystal structure of $\beta$ selenium. Acta Cryst. 6, 71-75 (1953).

18. Parthasarathy, G. \& Holzapfel, W. B. Structural phase transitions and equations of state for selenium under pressure. Phys. Rev. B 38, 10105-10108 (1988).

19. Akahama, Y., Kobayashi, M. \& Kawamura, H. Structural studies of pressure-induced phase transitions in selenium up to 150 GPa. Phys. Rev. B 47, 20-26 (1993).

20. Akahama, Y., Kobayashi, M. \& Kawamura, H. High-pressure phase transition to $\beta$-polonium type structure in selenium. Solid State Commun. 83, 273-276 (1992).

21. Luo, H., Greene, R. G. \& Ruoff, A. L. $\beta$-Po phase of sulfur at 162 GPa: X-ray diffraction study to 212 GPa. Phys. Rev. Lett. 71, 2943-2946 (1993).

22. Warren, B. E. \& Burwell, J. T. The structure of rhombic sulphur. J. Chem. Phys. 3, 6-8 (1935).

23. Akahama, Y., Kobayashi, M. \& Kawamura, H. Pressure-induced structural phase transition in sulfur at 83 GPa. Phys. Rev. B 48, 6862-6864 (1993).

24. Degtyareva, O., Gregoryanz, E., Somayazulu, M., Mao, H. K. \& Hemley, R. J. Crystal structure of the superconducting phases of S and Se. Phys. Rev. B 71, 214104 (2005).

25. Einaga, M. et al. Crystal structure of $200 \mathrm{~K}$-superconducting phase of sulfur hydride system. arXiv 1509.03156v1 (2015).

26. Struzhkin, V. V., Hemley, R. J., Mao, H. K. \& Timofeev, Y. A. Superconductivity at 10-17 K in compressed sulphur. Nature 390, 382-384 (1997).

27. Akahama, Y., Kobayashi, M. \& Kawamura, H. Pressure induced superconductivity and phase transition in selenium and tellurium. Solid State Commun. 84, 803-806 (1992).

28. Drozdov, A. P., Eremets, M. I., Troyan, I. A., Ksenofonto, V. \& Shylin, S. I. Conventional superconductivity at 203 kelvin at high pressures in the sulfur hydride system. Nature 525, 73-76 (2015).

29. Speziale, S., Zha, C. S., Duffy, T. S., Hemley, R. J. \& Mao, H. K. Quasi-hydrostatic compression of magnesium oxide to 52 GPa: Implications for the pressure-volume-temperature equation of state. J. Geophys. Res. 106, 515-528 (2001).

30. Li, B., Woody, K. \& Kung, J. Elasticity of MgO to $11 \mathrm{GPa}$ with an independent absolute pressure scale: Implications for pressure calibration. J. Geophys. Res. 111, B11206 (2006).

31. Fei, Y. Effects of temperature and composition on the bulk modulus of (Mg,Fe)O. Am. Mineral. 84, 272-276 (1999).

32. Dewaele, A., Fiquet, G., Andrault, D. \& Hausermann, D. P-V-T equation of state of periclase from synchrotron radiation measurements, J. Geophys. Res. 105, 2869-2877 (2000).

33. Utsumi, W., Weidner, D. J. \& Liebermann, R. C. Properties of Earth and Planetary Materials at High Pressure and Temperature (eds Manghnani, M. H. \& Yagi, T.) pp. 327-333 (American Geophysical Union, 1998).

34. Hirose, K., Sata, N., Komabayashi, T. \& Ohishi, Y. Simultaneous volume measurements of Au and MgO to $140 \mathrm{GPa}$ and thermal equation of state of Au based on the MgO pressure scale. Phys. Earth Planet. Interiors. 167, 149-154 (2008).

35. Jacobsen, S. D. et al. Compression of single-crystal magnesium oxide to $118 \mathrm{GPa}$ and a ruby pressure gauge for helium pressure media. Am. Mineral. 93, 1823-1828 (2008).

36. Utsumi, W., Funamori, N. \& Yagi, T. Thermal expansivity of $\mathrm{MgSiO}_{3}$ perovskite under high pressures up to $20 \mathrm{GPa}$. Geophys. Res. Lett. 22, 1005-1008 (1995). 
37. Fiquet, G. et al. P-V-T equation of state of $\mathrm{MgSiO}_{3}$ perovskite. Phys. Earth Planet. Interiors. 105, 21-31 (1998).

38. Saxena, S. K., Dubrovinsky, L. S., Tutti, F. \& Bihan, T. L. Equation of state of $\mathrm{MgSiO}_{3}$ with the perovskite structure based on experimental measurement. Am. Mineral. 84, 226-232 (1999).

39. Fiquet, G., Dewaele, A., Andrault, D., Kunz, M. \& Bihan, T. L. Thermoelastic properties and crystal structure of $\mathrm{MgSiO}_{3}$ perovskite at lower mantle pressure and temperature conditions. Geophys. Res. Lett. 27, 21-24 (2000).

40. Vanpeteghem, C. B., Zhao, J., Angel, R. J., Ross, N. L. \& Bolfan-Casanova, N. Crystal structure and equation of state of $\mathrm{MgSiO}_{3}$ perovskite. Geophys. Res. Lett. 33, L03306 (2006).

41. Sakai, T., Dekura, H. \& Hirao, N. Experimental and theoretical thermal equations of state of $\mathrm{MgSiO}_{3}$ post-perovskite at multimegabar pressures. Sci. Rep. 6, 22652 (2016).

42. Anderson, D. L. Theory of the Earth. Ch. 5, 79-102 (Blackwell Scientific Publications, 1989).

43. Crowhurst, J. C., Brown, J. M., Goncharov, A. F. \& Jacobsen, S. D. Elasticity of (Mg,Fe)O Through the Spin Transition of Iron in the Lower Mantle. Science 319, 451-453 (2008).

44. Zhang, L. et al. Disproportionation of ( $\mathrm{Mg}, \mathrm{Fe}) \mathrm{SiO}_{3}$ perovskite in Earth's deep lower mantle. Science 344, 877-882 (2016).

45. Rodges, P. A. Pressure-volume-temperature relationships for polymeric liquids: A review of equations of state and their characteristic parameters for 56 polymers. J. Appl. Polym. Sci. 48, 1061-1080 (1993).

46. Chen, C. T., Fine, R. A. \& Millero, F. J. The equation of state of pure water determined from sound speeds. J. Chem. Phys. 66, 2142-2144 (1977).

47. Blöchl, P. E. Projector augmented-wave method. Phys. Rev. B 50, 17953-17979 (1994).

48. Kresse, G. \& Joubert, D. From ultrasoft pseudopotentials to the projector augmented-wave method. Phys. Rev. B 59, 1758-1775 (1999).

49. Kresse, G. \& Furthmüller, J. Efficient iterative schemes for ab initio total-energy calculations using a plane-wave basis set. Phys. Rev. B 54, 11169-11186 (1996).

50. Perdew, J. P., Burke, K. \& Ernzerhof, M. Generalized Gradient Approximation Made Simple. Phys. Rev. Lett. 77, 3865-3868 (1996).

51. Anderson, M. S. \& Swenson, C. A. Experimental compressions for normal hydrogen and normal deuterium to $25 \mathrm{kbar}$ at $4.2 \mathrm{~K}$. Phys. Rev. B 10, 5184-5191 (1974).

52. Wanner, R. \& Meyer, H. Velocity of sound in solid hexagonal close-packed $\mathrm{H}_{2}$ and $\mathrm{D}_{2}$. J. Low. Temp. Phys. 11, 715-744 (1973).

\section{Acknowledgements}

This research used resources of the National Energy Research Scientific Computing Center, a DOE Office of Science User Facility supported by the Office of Science of the U.S. Department of Energy under Contract No. DE-AC02-05CH11231. MHW thanks Prof. Jerry L. Whitten for invaluable discussion concerning the qualitative meaning of the isothermal EOS, Eq. 2.

\section{Author Contributions}

This work was conceived by M.-H.W. and J.K., and E.E.G. carried out all DFT calculations. The empirical EOS was realized by M.-H.W. and E.E.G. while analyzing the computational results. Further testing of the EOS was designed by M.-H.W. and J.K., and E.E.G. carried out the analyses. All authors participated in the discussion of the draft written by M.-H.W.

\section{Additional Information}

Supplementary information accompanies this paper at http://www.nature.com/srep

Competing financial interests: The authors declare no competing financial interests.

How to cite this article: Gordon, E. E. et al. Condensed-matter equation of states covering a wide region of pressure studied experimentally. Sci. Rep. 6, 39212; doi: 10.1038/srep39212 (2016).

Publisher's note: Springer Nature remains neutral with regard to jurisdictional claims in published maps and institutional affiliations.

(c) (i) This work is licensed under a Creative Commons Attribution 4.0 International License. The images or other third party material in this article are included in the article's Creative Commons license, unless indicated otherwise in the credit line; if the material is not included under the Creative Commons license, users will need to obtain permission from the license holder to reproduce the material. To view a copy of this license, visit http://creativecommons.org/licenses/by/4.0/

(C) The Author(s) 2016 


\section{SCIENTIFIC REP RTS}

\section{OPEN Erratum: Condensed-matter equation of states covering a wide region of pressure studied experimentally}

\section{Elijah E. Gordon, Jürgen Köhler \& Myung-Hwan Whangbo}

Scientific Reports 6:39212; doi: 10.1038/srep39212; published online 15 December 2016; updated on 12 January 2017

The original version of this Article contained typographical errors.

In the Abstract,

"Here we show that the simple empirical EOS, $\mathrm{P}=\alpha^{1}(\mathrm{PV})+\alpha^{2}(\mathrm{PV})^{2}+\alpha^{3}(\mathrm{PV})^{3}$, in which the pressure $\mathrm{P}$ is indirectly related to the volume $\mathrm{V}$ through a cubic polynomial of the energy term $\mathrm{PV}$ with three fitting parameters $\alpha^{1}-\alpha^{3}$, provides accurate descriptions for the P-vs- $V$ data of condensed matter in a wide region of pressure studied experimentally even in the presence of phase transitions".

now reads:

"Here we show that the simple empirical EOS, $P=\alpha_{1}(P V)+\alpha_{2}(P V)^{2}+\alpha_{3}(P V)^{3}$, in which the pressure $P$ is indirectly related to the volume $\mathrm{V}$ through a cubic polynomial of the energy term $\mathrm{PV}$ with three fitting parameters $\alpha_{1}-\alpha_{3}$, provides accurate descriptions for the P-vs- $\mathrm{V}$ data of condensed matter in a wide region of pressure studied experimentally even in the presence of phase transitions".

In the Results section under subheading 'Applicability to other condensed matter',

"To establish this point, we examine the experimental $P$-vs- $V$ data for various solid-state condensed matter listed in Table 1, which include the elemental $\mathrm{Sn}$, the transition-metals $\mathrm{Au}$ and $\mathrm{Cu}$, the alkali halides $\mathrm{LiF}, \mathrm{NaF}, \mathrm{NaCl}$ and $\mathrm{CsCl}$, ice VII, the oxides $\mathrm{MgO}$ and $\mathrm{MgSiO}_{3}$, the noble gases $\mathrm{Ar}, \mathrm{Kr}$ and $\mathrm{Xe}$, as well as molecular hydrogen $\mathrm{H}_{2} \mathrm{D}_{2}$ ".

now reads:

"To establish this point, we examine the experimental $P$-vs- $V$ data for various solid-state condensed matter listed in Table 1, which include the elemental Sn, the transition-metals $\mathrm{Au}$ and $\mathrm{Cu}$, the alkali halides $\mathrm{LiF}, \mathrm{NaF}, \mathrm{NaCl}$ and $\mathrm{CsCl}$, ice VII, the oxides $\mathrm{MgO}$ and $\mathrm{MgSiO}_{3}$, the noble gases $\mathrm{Ar}, \mathrm{Kr}$ and $\mathrm{Xe}$, as well as molecular hydrogen $\mathrm{H}_{2}$ and $\mathrm{D}_{2}$.

These errors have now been corrected in the PDF and HTML versions of the Article.

(c) (i) This work is licensed under a Creative Commons Attribution 4.0 International License. The images or other third party material in this article are included in the article's Creative Commons license, unless indicated otherwise in the credit line; if the material is not included under the Creative Commons license, users will need to obtain permission from the license holder to reproduce the material. To view a copy of this license, visit http://creativecommons.org/licenses/by/4.0/

(c) The Author(s) 2017 